\title{
SIMILARITY OF PARTS TO THE WHOLE FOR CERTAIN MULTIPLICATION OPERATORS
}

\author{
PAUL S. BOURDON
}

\begin{abstract}
We show that the Bergman shift $B$, multiplication by $z$ on the Bergman space $A^{2}$, is similar to its part $\left.B\right|_{N}$ if and only if $N=\varphi A^{2}$, where $\varphi$ is a finite product of interpolating Blaschke products. In addition, we show that $B$ is not unitarily equivalent to any of its parts. For the analytic Toeplitz operator $T_{f}$ on $H^{2}$, we obtain that $T_{f}$ is similar to each of its parts if and only if $T_{f}$ is unitarily equivalent to each of its parts if and only if $f$ is a weak-star generator of $H^{\infty}$.
\end{abstract}

1. Introduction. Let $T$ be a bounded linear operator on a Hilbert space $H$. If $M$ is a closed subspace of $H$ such that $T M \subset M$, then $M$ is said to be invariant for $T$. The restriction of $T$ to one of its nonzero invariant subspaces is said to be a part of $T$. In this paper, we consider the problem of determining which parts of the Bergman shift are similar to the Bergman shift and the problem of determining when an analytic Toeplitz operator is similar to each of its parts.

The operators $T_{1}: H_{1} \rightarrow H_{1}$ and $T_{2}: H_{2} \rightarrow H_{2}$ are similar, written $T_{1} \approx T_{2}$, if there is an invertible operator $S: H_{1} \rightarrow H_{2}$ such that $T_{2} S=S T_{1}$. If $S$ is unitary, then $T_{1}$ and $T_{2}$ are said to be unitarily equivalent, written $T_{1} \cong T_{2}$.

Recall that the Bergman space $A^{2}$ is the Hilbert space of those functions $f$ analytic on the open unit disk $U$ which satisfy

$$
\|f\|_{A^{2}}^{2}=\frac{1}{\pi} \int_{0}^{2 \pi} \int_{0}^{1}\left|f\left(r e^{i \theta}\right)\right|^{2} r d r d \theta<\infty .
$$

In the following section, we show that the Bergman shift $B$, multiplication by $z$ on $A^{2}$, is similar to its part $\left.B\right|_{N}$ if and only if $N=\varphi A^{2}$, where $\varphi$ is a finite product of interpolating Blaschke products. We also show that $B$ is not unitarily equivalent to any of its parts. This contrasts sharply with the situation for the unweighted shift, multiplication by $z, T_{z}$, on the Hardy space of $U, H^{2}$. For any $f \in H^{\infty}$, let $T_{f}: H^{2} \rightarrow H^{2}$ be the analytic Toeplitz operator with symbol $f$; hence, $\left(T_{f} h\right)(z)=f(z) h(z)$.

Proposition. $T_{z}: H^{2} \rightarrow H^{2}$ is unitarily equivalent to each of its parts.

PROOF. By Beurling's Theorem [1] each nonzero invariant subspace for $T_{z}$ on $H^{2}$ has the form $\varphi H^{2}$ for some inner function $\varphi$. The operator $T_{\varphi}: H^{2} \rightarrow \varphi H^{2}$ is unitary (an onto isometry) and $\left.T_{z}\right|_{\varphi H^{2}} T_{\varphi}=T_{\varphi} T_{z}$. Hence, the unweighted shift is unitarily equivalent to each of its parts.

It is natural to ask when an analytic Toeplitz operator shares with the unweighted shift the property that it is unitarily equivalent to each of its parts. In fact, Wang

Received by the editors January 27, 1986.

1980 Mathematics Subject Classification (1985 Revision). Primary 47B35, 47B37. 
and Stampli [9] have raised the following question: If $f \in H^{\infty}$ and $T_{f}$ is unitarily equivalent to each of its parts, is $f$ a weak-star generator of $H^{\infty}$; i.e., are the polynomials in $f$ weak-star dense in $H^{\infty}$ ?

In $\S 3$, we answer the question of Wang and Stampfli in the affirmative by proving the following theorem.

THEOREM 3.1. The following are equivalent.

(a) $f$ is a weak-star generator of $H^{\infty}$.

(b) $T_{f}$ has the same invariant subspaces as $T_{z}$.

(c) $T_{f}$ is unitarily equivalent to each of its parts.

(d) $T_{f}$ is similar to each of its parts.

The equivalence of (a) and (b) was established by Sarason [7]. That (b) implies (c) is an easy consequence of Beurling's Theorem. What appears to be new is that (c) and (d) are equivalent and that either implies (a).

2. Parts of the Bergman shift similar to the Bergman shift. Suppose $T: H \rightarrow H$ is similar to its part $\left.T\right|_{N}$ so that there is an invertible operator $S: H \rightarrow$ $N$ such that $\left.T\right|_{N} S=S T$. Note that $S$ viewed as an operator on $H$ commutes with $T$ and is bounded below. Recall that an operator $S$ on $H$ is bounded below if there is a constant $\delta>0$ such that $\|S f\| \geq \delta\|f\|$ for all $f \in H$; hence, $S$ is bounded below if and only if it is 1-1 with closed range.

Proposition 2.1. If $S: H \rightarrow H$ is bounded below and commutes with $T$, then $\operatorname{Ran} S$ is invariant for $T$ and $\left.T\right|_{\operatorname{Ran} S} \approx T$. If $S$ is an isometry which commutes with $T$, then $\left.T\right|_{\operatorname{Ran} S} \cong T$.

Proof. Since $S$ is bounded below, $\operatorname{Ran} S=S H$ is closed; and since $T$ commutes with $S, T S H=S T H \subset S H$. Thus $\operatorname{Ran} S$ is invariant for $T$. Now, $S: H \rightarrow \operatorname{Ran} S$ is invertible and $\left.T\right|_{\operatorname{Ran} S} S=S T$; hence, $\left.T \approx T\right|_{\operatorname{Ran} S}$. If $S$ is an isometry, the map $S: H \rightarrow \operatorname{Ran} S$ is unitary.

For any $f \in H^{\infty}$ define $M_{f}: A^{2} \rightarrow A^{2}$ to be the operator of multiplication by $f$ on $A^{2}$. Let $C=\left\{\varphi \in H^{\infty}: \varphi A^{2}\right.$ is a nonzeroclosed subspace of $\left.A^{2}\right\}=\left\{\varphi \in H^{\infty}\right.$ : $M_{\varphi}$ is bounded below $\}$.

Proposition 2.2. The Bergman shift $M_{z}: A^{2} \rightarrow A^{2}$ is similar to its part $\left.M_{z}\right|_{N}: N \rightarrow N$ if and only if $N=\varphi A^{2}$ for some $\varphi \in C$.

PROOF. If $\varphi \in C$, then $M_{\varphi}$ is bounded below and $M_{z}$ restricted to $\operatorname{Ran} M_{\varphi}=$ $\varphi A^{2}$ is similar to $M_{z}$ by Proposition 2.1. Conversely, if $\left.M_{z}\right|_{N}$ is similar to $M_{z}$, there is an invertible operator $S: A^{2} \rightarrow N$ such that $\left.M_{z}\right|_{N} S=S M_{z}$. Since the operator $S$ commutes with $M_{z}$, there is a $\varphi \in H^{\infty}$ such that $S=M_{\varphi}$ [8, Theorem 3]. Now,

$$
N=\operatorname{Ran} S=\operatorname{Ran} M_{\varphi}=\varphi A^{2}
$$

and $\varphi \in C$ since $N=\varphi A^{2}$ is closed and nonzero.

Thus the problem of finding those parts of $M_{z}: A^{2} \rightarrow A^{2}$ which are similar to $M_{z}$ is reduced to that of finding those $\varphi \in H^{\infty}$ for which $M_{\varphi}$ is bounded below. The following result of D. Leucking [5, Corollary 1] characterizes those $\varphi$ for which $M_{\varphi}$ is bounded below on $A^{2}$. 
THEOREM 2.3 (LEUCKING). Let $h \in H^{\infty}$. The operator $M_{h}: A^{2} \rightarrow A^{2}$ is bounded below if and only if there are positive numbers $r$ and $\delta$ such that $G=$ $\{z:|h(z)|>r\}$ satisfies $m(G \cap D) \geq \delta m(U \cap D)$ for all disks $D$ with center on the circle $|z|=1$. (Here, $m$ represents planar Lebesgue measure.)

We give another characterization of those $h \in H^{\infty}$ for which $M_{h}$ is bounded below on $A^{2}$. The characterization is based on the following result of McDonald and Sundberg [6, Proposition 22] (cf. also Horowitz [4, Theorem 2]).

THEOREM 2.4 (MCDONALD-SUNDBERG). Let $\varphi$ be an inner function. $M_{\varphi}$ : $A^{2} \rightarrow A^{2}$ is bounded below if and only if $\varphi$ is a finite product of interpolating Blaschke products (f.p.i.b.p.).

Any function $h \in H^{\infty}$ has the factorization $h=P S F$, where $P$ is a Blaschke product, $S$ is a singular inner function, and $F$ is an outer function in $H^{\infty}$. A consequence of Beurling's Theorem is that $\{p(z) F: p$ is a polynomial $\}$ is dense in $H^{2}$ for any outer function $F \in H^{2}$. Since convergence in $H^{2}$ implies convergence in $A^{2}$ and since $H^{2}$ is contained in $A^{2}$ as a dense subset (the polynomials are dense in $A^{2}$ ), we must have that $\{p(z) F: p$ is a polynomial $\}$ is dense in $A^{2}$ for any outer function $F \in H^{2}$. In particular, the operator $M_{F}: A^{2} \rightarrow A^{2}$ has dense range provided $F \in H^{\infty}$ is outer.

COROLLARY 2.5. Let $h \in H^{\infty}$. The operator $M_{h}: A^{2} \rightarrow A^{2}$ is bounded below if and only if $h=\varphi F$, where $F, 1 / F \in H^{\infty}$ and where $\varphi$ is a f.p.i.b.p. In this case $\operatorname{Ran} M_{h}=\varphi A^{2}$.

ProOF. If $h=\varphi F$ where $F, 1 / F \in H^{\infty}$ and $\varphi$ is a f.p.i.b.p., then $\operatorname{Ran} M_{h}=$ $\varphi F A^{2}=\varphi A^{2}$ is closed by Theorem 2.4. Hence, $M_{h}$ is bounded below.

Now suppose that $M_{h}: A^{2} \rightarrow A^{2}$ is bounded below. Let $h=\varphi S F$, where $\varphi$ is a Blaschke product, $S$ is a singular inner function, and $F$ is an outer function in $H^{\infty}$. Since $M_{h}=M_{\varphi S} M_{F}=M_{F} M_{\varphi S}$, both $M_{F}$ and $M_{\varphi S}$ must be bounded below. By Theorem 2.4, $S \equiv 1$ and $\varphi$ is a f.p.i.b.p. Now, in addition to being bounded below, $M_{F}$ has dense range since $F \in H^{\infty}$ is outer. It follows that $M_{F}$ is invertible, and hence, $F, 1 / F \in H^{\infty}$.

The following theorem is an immediate consequence of Proposition 2.2 and Corollary 2.5 .

THEOREM 2.6. The Bergman shift $B: A^{2} \rightarrow A^{2}$ is similar to its part $\left.B\right|_{N}$ if and only if $N=\varphi A^{2}$, where $\varphi$ is a f.p.i.b.p.

It is clear from the proof of Proposition 2.2 that the problem of determining which parts of $B$ are unitarily equivalent to $B$ is equivalent to that of determining which of the operators $M_{h}: A^{2} \rightarrow A^{2}$ are isometries. However, none of these operators other than $M_{1}$ is an isometry.

Proposition 2.7. The Bergman shift is not unitarily equivalent to any of its (proper) parts.

Proof. Suppse $\left.B \cong B\right|_{N}$. By the proof of Proposition $2.2, N=\operatorname{Ran} M_{h}$ for some isometry $M_{h}: A^{2} \rightarrow A^{2}\left(h \in H^{\infty}\right)$. Since $\|1\|_{A^{2}}=1$ and $M_{h}$ is an isometry, $\|h\|_{A^{2}}=1$. Now, for any $z \in U,|h(z)| \leq\left\|M_{h}\right\|=1[3$, Lemma 11]. By the maximum modulus theorem, $h(z) \equiv 1$ or $|h(z)|<1$ for all $z \in U$. If $h \equiv 1$, then 
$N=\operatorname{Ran} M_{1}=A^{2}$ and $\left.B\right|_{N}$ is the "whole" Bergman shift. If $|h(z)|<1$ for all $z \in U$, then

$$
\|h\|_{A^{2}}^{2}=\frac{1}{\pi} \int_{0}^{2 \pi} \int_{0}^{1}\left|h\left(r e^{i \theta}\right)\right|^{2} r d r d \theta<1,
$$

a contradiction.

REMARKS. 1. Proposition 2.2 stated for the Bergman shift has an analogue for any shift operator $M_{z}: H^{2}(\beta) \rightarrow H^{2}(\beta)$ with $C$ replaced by $C_{\beta}=\{\varphi \in$ $H^{\infty}(\beta): M_{\varphi}: H^{2}(\beta) \rightarrow H^{2}(\beta)$ is bounded below (cf. [8] for notation).

2. Theorems $2.3,2.4$, and Corollary 2.5 remain valid if $A^{2}$ is replaced by any member $A^{p}(p>0)$ of the Bergman family of spaces.

3. The author was led to consider the question of what parts of $B$ are similar to $B$ as a first step in solving the problem of grouping parts of $B$ into equivalence classes under similarity (or unitary equivalence). This problem was raised by $\mathrm{H}$. Bercovici and was related to the author by C. Cowen. It is easy to see that $\left.\left.B\right|_{N_{2}} \approx B\right|_{N_{1}}$ if $N_{2}=\varphi N_{1}$ for $\varphi$ a f.p.i.b.p., but it seems likely that there are other parts of $B$ similar to $\left.B\right|_{N_{1}}$.

3. Uniform operators. Adopting terminology used by Wang and Stampli in [9], we say that an operator $T$ on a separable infinite dimensional Hilbert space $H$ is uniform provided $T$ is unitarily equivalent to $\left.T\right|_{M}$ for every infinite dimensional subspace $M \subset H$ invariant for $T$. Hence, for example, the unweighted shift is uniform. In [9], Wang and Stampfli obtain a general representation theorem for uniform operators. Using the representation theorem, they show that a uniform operator which has an invariant subspace of finite codimension must be an analytic Toeplitz operator [9, Theorem 5]. If $f \in H^{\infty}$ is nonconstant, then it is easy to see that any nonzero subspace invariant for $T_{f}$ must be infinite dimensional. Hence, Theorem 3.1 below and the result of Wang and Stampfli combine to show that a uniform operator which has an invariant subspace of finite codimension must be either a constant multiple of the identity or an analytic Toeplitz operator whose symbol is a weak-star generator of $H^{\infty}$.

THEOREM 3.1. The following are equivalent.

(a) $f$ is a weak-star generator of $H^{\infty}$.

(b) $T_{f}$ has the same invariant subspaces as $T_{z}$.

(c) $T_{f}$ is unitarily equivalent to each of its parts.

(d) $T_{f}$ is similar to each of its parts.

Proof. (a) $\Leftrightarrow$ (b) Sarason [7].

(b) $\Rightarrow$ (c). Each nonzero invariant subspace for $T_{f}$ has the form $\varphi H^{2}$ for some inner function $\varphi$ (Beurling's Theorem). Now, $T_{\varphi}: H^{2} \rightarrow \varphi H^{2}$ is unitary and $\left.T_{f}\right|_{\varphi H^{2}} T_{\varphi}=T_{\varphi} T_{f}$. Hence, $T_{f}$ is unitarily equivalent to each of its parts.

(c) $\Rightarrow$ (d). Trivial.

(d) $\Rightarrow$ (b). Since any subspace invariant for $T_{z}$ is easily seen to be invariant for $T_{f}$, what we must show is that any subspace $M \subset H^{2}$ invariant for $T_{f}$ is also invariant for $T_{z}$.

Let $\langle f\rangle$ be the closed subspace of $H^{2}$ generated by $\left\{1, f, f^{2}, \ldots\right\} .\langle f\rangle$ is invariant for $T_{f}$ and $\left.T_{f}\right|_{\langle f\rangle}$ is cyclic with cyclic vector 1 . Since $\left.T_{f}\right|_{\langle f\rangle}$ is similar to $T_{f}, T_{f}$ is cyclic with, say, cyclic vector $g$. 
Let $M \subset H^{2}$ be an arbitrary invariant subspace for $T_{f}$. We need to show that $z M \subset M$. Since $T_{f}$ is similar to $\left.T_{f}\right|_{M}$, there is an invertible operator $S: H^{2} \rightarrow M$ such that $\left.T_{f}\right|_{M} S=S T_{f}$. Since $g$ is cyclic for $T_{f}, S g$ is cyclic for $\left.T_{f}\right|_{M}$. Claim $z S g \in M$.

Singe $g$ is cyclic for $T_{f}$, there is a sequence $\left\{q_{n}\right\}$ of polynomials such that $\left\|q_{n}\left(T_{f}\right) g-z g\right\| \rightarrow 0$. Since convergence in $H^{2}$ implies pointwise convergence on the unit disk $U, q_{n}(f) \rightarrow z$ pointwise on $U$. Now,

$$
\left\|q_{n}\left(\left.T_{f}\right|_{M}\right) S g-S(z g)\right\|=\left\|S q_{n}\left(T_{f}\right) g-S(z g)\right\| \rightarrow 0
$$

by the continuity of $S$. It follows that $q_{n}(f) S g \rightarrow S(z g)$ pointwise on $U$, but $q_{n}(f) \rightarrow z$ pointwise. Hence, $z S g=S(z g) \in M$.

We now see that the dense subspace of $M,\{p(f) S g: p$ is a polynomial $\}$, is mapped into $M$ under $T_{z}$. It follows that $z M \subset M$.

REMARKS. 1. Results related to those of this section but concerning normal operators and their nonnormal parts may be found in $[2, \S 3]$.

2. The operators $T_{1}: H_{1} \rightarrow H_{1}$ and $T_{2}: H_{2} \rightarrow H_{2}$ are quasisimilar if there are injective operators with dense range $X_{12}: H_{2} \rightarrow H_{1}$ and $X_{12}: H_{1} \rightarrow H_{2}$ such that $X_{12} T_{2}=T_{1} X_{12}$ and $X_{21} T_{1}=T_{2} X_{21}$. It is not difficult to see how to modify the proof of Theorem $3.1,(\mathrm{~d}) \Rightarrow(\mathrm{b})$, to show that if $T_{f}$ is quasisimilar to each of its parts, then $T_{f}$ and $T_{z}$ have the same invariant subspaces. Hence " $T_{f}$ is quasisimilar to each of its parts" may be added to the list of equivalent conditions given in Theorem 3.1 .

\section{REFERENCES}

1. A. Beurling, On two problems concerning linear transformations in Hilbert space, Acta Math. 81 (1949), 239-255.

2. J. Conway, On quasisimilarity for subnormal operators, Illinois J. Math. 24 (1980), 689-702.

3. P. Duren, B. Romberg, and A. Shields, Linear functionals on $H^{p}$ spaces with $0<p<1$, J. Reine Angew. Math. 238 (1969), 32-60.

4. C. Horowitz, Factorization theorems for functions in the Bergman spaces, Duke Math. J. 44 (1977), 201-213.

5. D. Leucking, Inequalities on Bergman spaces, Illinois J. Math. 25 (1981), 1-11.

6. G. McDonald and C. Sundberg, Toeplitz operators on the disc, Indiana Univ. Math. J. 28 (1979), 595-611.

7. D. Sarason, Invariant subspaces and unstarred operator algebras, Pacific J. Math. 17 (1966), 511517.

8. A. Shields, Weighted shift operators and analytic function theory, Topics in Operator Theory (C. Pearcy, ed.), Math. Surveys, no. 13, Amer. Math. Soc., Providence, R.I., 1974, pp. 49-128.

9. H. Wang and J. Stampfli, Uniform operators, Trans. Amer. Math. Soc. 289 (1985), 163-169.

Department of Mathematics, Michigan State University, East lansing, MICHIGAN 48824 